\title{
Lateral diffusion of single poly(ethylene oxide) chains on the surfaces of glassy and molten polymer films
}

Matthew Mears, ${ }^{1}$ Zhenyu J. Zhang, ${ }^{1}$ Ryan C. D. Jackson, ${ }^{1}$ Yuchen Si, ${ }^{1}$ Tigerlily J. B. Bradford, ${ }^{1}$ John M. Torkelson, ${ }^{2}$ and Mark Geoghegan ${ }^{1, *}$

1. Department of Physics and Astronomy, University of Sheffield, Sheffield S3 7RH, UK

2. Department of Chemical and Biological Engineering and Department of Materials Science and Engineering, Northwestern University, Evanston, IL 60208 USA

Fluorescence correlation spectroscopy was used to show that the temperature-dependent diffusion coefficient of poly(ethylene oxide) (PEO) adsorbed on polystyrene and different poly(alkyl methacrylate) (PAMA) films in aqueous solution exhibited a maximum close to (but below) the surface glass transition temperature, $T_{\mathrm{gs}}$ of the film. This elevated diffusion was observed over a small range of temperature below $T_{\mathrm{gs}}$ for these surfaces, and at other temperatures the diffusion was similar to that on silicon, although the diffusion coefficient for PEO on polystyrene at temperatures above $T_{\mathrm{gs}}$ did not completely decrease to that on silicon, in contrast to the PAMA surfaces. It is concluded that the enhanced surface mobility of the films near the surface glass transition temperature induces conformational changes in the adsorbed PEO. The origin of this narrow and dramatic increase in diffusion coefficient is not clear, but it is proposed that it is caused by a coupling of a dominant capillary mode in the liquid surface layer with the polymer. Friction force microscopy experiments also demonstrate an unexpected increase in friction at the same temperature as the increase in diffusion coefficient.

\section{INTRODUCTION}

Molecular theory underpinning the physics of the glass transition in polymers is still lacking, but the dramatic effect of confinement on the glass transition has been known for some time to play a key role. ${ }^{1-4}$ The original experiments on thin films of polystyrene ${ }^{5}$ and, shortly after, poly(methyl methacrylate) (PMMA) ${ }^{6}$ demonstrated that there was a significant reduction in the glass transition temperature $\left(T_{\mathrm{g}}\right)$ with film thickness, but could provide only an empirical description of their behavior. Despite the difficulties that traditional semi-empirical free volume theories have experienced, the concept of free volume as a means to explain the glass transition of confined polymer films has remained remarkably robust. ${ }^{3,4,7,8}$

An important early question was related to whether confinement or the free surface of the film was responsible for the depressed glass transition. Early experiments on dewetting were explained by arguing that thinner films were less dense than in the bulk and consequently the effective glass transition temperature was reduced. ${ }^{9}$ These arguments contradicted a popular idea that chain conformations were unperturbed by a surface, ${ }^{10,11}$ but it has subsequently been accepted that polymers near the surface of a film do not have the same conformation as those deeper into the film. ${ }^{12-14}$ Later experiments were able to identify that the glass transition increased with distance from the surface of the film, ${ }^{15}$ although this effect was strongly dependent upon the chemical nature of the polymer beneath the surface layer. ${ }^{16}$ It has since been argued that a discrete liquid surface layer occurs below the bulk $T_{\mathrm{g}}$ for both entangled and unentangled films. ${ }^{17}$

On the one hand, a surface presents itself as an interface to a semi-infinite reservoir of free volume, ${ }^{18}$ which aids relaxation of the chains but on the other hand, the surface perturbs the film itself, increasing the segmental mobility in its vicinity. ${ }^{19}$ The surface therefore plays a profound role in the depressed glass transition of polymer films. The glass transition in a fluid environment is less understood, although work on polystyrene films and nanoparticles suggests that an aqueous interface with the film does not noticeably change the depression in the glass transition temperature. ${ }^{20}$

The lateral diffusion of polymers on surfaces is an ideal means of testing for changes in the behavior of that surface because it strongly depends on the nature of the adsorption. ${ }^{21} \mathrm{~A}$ polymer that is fully adsorbed in a 'pancake' configuration ${ }^{22}$ on the surface is constrained and exhibits less movement than one that has fewer contact points on the surface. ${ }^{23}$ The diffusion of $\mathrm{PEO}$ on surfaces is a function of other parameters such as 
the concentration of adsorbed polymer ${ }^{24-27}$ or the presence of topographic constraints. ${ }^{28,29}$ All of these experiments concern the diffusion at the solid-liquid interface. Little is known of how a polymer diffuses at the aqueous interface with a molten polymer film, which is subject to capillary waves, which themselves are influenced by the surface melting associated with the glass transition. ${ }^{30}$

In this work, single poly(ethylene oxide) (PEO) chains are allowed to diffuse in an aqueous environment on polymer surfaces as a function of temperature. The surfaces comprised polystyrene and different poly(alkyl methacrylate) homopolymer and blend films. It is proposed that there is a coupling of the polymer to the surface of the film approximately $10 \mathrm{~K}$ below the surface glass transition, which causes a large increase in the surface diffusion of the PEO over a narrow temperature range. A similar effect is observed using friction force microscopy.

\section{EXPERIMENTAL}

\section{A. Polymer films}

Silicon substrates (Prolog Semicor, Ukraine) were cleaved into approximately $1 \mathrm{~cm}^{2}$ sections, sonicated in chloroform and then toluene for $\sim 20 \mathrm{~min}$ in each, and cleaned for $\sim 15 \mathrm{~min}$ in an oxygen plasma. The cleaned substrates were then immediately coated with the relevant polymer by spin coating. Solutions of $5 \% \mathrm{w} / \mathrm{v}$ poly(alkyl methacrylate) or polystyrene in toluene were used and spin speeds ranging from 2000 to $4000 \mathrm{rpm}$ allowed control of the resulting film thickness to between approximately $250 \mathrm{~nm}$ and $350 \mathrm{~nm}$.

The polymers used for the films were PMMA (mass averaged molar mass, $M=120 \mathrm{kDa}$ and dispersity, $D=$ 2.0), PEMA $(M=250 \mathrm{kDa}$ and $D=2.3)$, PPMA $(M=150 \mathrm{kDa}$ and $D=2.4)$, PBMA $(M=337 \mathrm{kDa}$ and $D$ $=2.1)$, and polystyrene $(M=222 \mathrm{kDa}$ and $D=1.02)$. All polymers were purchased from Sigma Aldrich and used as received.

The resulting films were incubated at $353 \mathrm{~K}$ for a minimum of $8 \mathrm{~h}$ to allow any residual solvent to leave the film. Homopolymer films were heated to $\sim 30 \mathrm{~K}$ above the surface glass transition temperature, $T_{\mathrm{gs}}$, and held at this temperature for at least $1 \mathrm{~h}$ in order to allow the polymer chains to relax toward an equilibrium state before cooling at a constant rate of $2 \mathrm{~K} \mathrm{~min}^{-1}$ to $\sim 30 \mathrm{~K}$ below $T_{\mathrm{gs}}$ before performing FCS experiments. Experiments were also performed using blends of PBMA and PMMA, which were heated to $393 \mathrm{~K}$ for at least $1 \mathrm{~h}$ to allow both polymer components of the film to relax and then cooled at $2 \mathrm{~K} \mathrm{~min}^{-1}$ to $\sim 280 \mathrm{~K}$. Low temperature ellipsometry measurements were performed with the aid of a liquid nitrogen flow-cooled chamber, but FCS measurements were limited to $\sim 278 \mathrm{~K}$ to avoid condensation, precluding measurements using PBMA homopolymer films.

\section{B. Ellipsometry measurements}

The thickness of the films was determined using an M-2000 spectroscopic ellipsometer (J. A. Woollam Co., Inc.). The film temperatures were controlled using a Linkam heating stage (Linkam Scientific Instruments Ltd, Surrey, UK) with a TMS94 heat controller and LNP-1 nitrogen flow control. The Linkam stage was calibrated using the boiling points of various solvents. An ellipsometry-specific sealed chamber (Linkam Scientific Instruments Ltd) with a nitrogen gas flow was used to minimize atmospheric effects for thickness measurements.

A Cauchy model was developed and used within the native software to fit the data and extract the thickness. The resulting temperature-thickness curves were subsequently analyzed using the pro Fit v6.1.16 (QuantumSoft, Switzerland) software package to determine the glass transition temperatures (Figure S1).

\section{Diffusion measurements}

FCS measurements were performed using an LSM510 AxioVert inverted confocal microscope with a ConfoCor2 FCS module (Carl Zeiss, Germany). Alignment and calibration of the optics were performed using $10 \mu \mathrm{M}$ fluorescein isothiocyanate in water, and diffusion measurements were performed with fluorescein-labeled $\operatorname{PEO}(M=20 \mathrm{kDa}$ and $D=1.2$, purchased from Scientific Polymer Products Inc, 
Ontario, USA and used as received). Fluorescein was excited using the $488 \mathrm{~nm}$ line of an argon laser, and fluorescence emission was collected through a 510-560 nm band-pass filter and recorded with an avalanche photodiode. The film temperatures were controlled using a Linkam heating stage (Linkam Scientific Instruments Ltd) with TMS94 heat controller and LNP-1 nitrogen flow control. A custom-built mount was used to invert and secure the heating stage onto the microscope platform for FCS measurements. A $\sim 5 \mathrm{nM}$ solution of fluorescein isothiocyanate-labeled PEO was prepared in water, and a small volume $(<10 \mu \mathrm{L})$ was placed onto the film before mounting onto the microscope stage. The droplet was confined between the polymer film and microscope objective lens, so evaporation was kept to a minimum. Little change in concentration was observed during the experiments. Experiments were performed on heating the samples but cooling the samples from an elevated temperature yielded identical results (Figure S3).

Accurate positioning of the confocal volume onto the surface of the film was critical in preventing the introduction of artefacts into the correlation curves, particularly because silicon substrates can act as a mirror and amplify noise as well as the signal. The confocal volume was first coarsely focused close to the surface using the on-screen image, after which z-scanning was performed in $100 \mathrm{~nm}$ steps from the bulk solution towards the coated surface. The position of the surface was determined by the peak signal-to-noise ratio from the automated z-positioning of the ConfoCor2 system. The surface diffusion signal was always significantly larger than that of bulk diffusion, and so was easy to identify. Typically, the number, $N$, of labelled PEO on the surface over all temperatures was $3<N<7$. There was little variation of $N$ with temperature. In order to eliminate any effect due to variations in temperature across the surface, the data were all obtained at the same point on the film at each temperature. After allowing 15 minutes equilibration time at each temperature, data were taken over a period of one hour. The long measurement times over a complete temperature scan did not cause any problems, as can be concluded from the heating and cooling scans (Figure S3).

Autocorrelation data were extracted from the native Zeiss software and fitted using the pro Fit software package with the autocorrelation function including both three- and two-dimensional components. ${ }^{31}$ The autocorrelation function is given by

$$
G(\tau)=1+\frac{1}{N}\left(\frac{(1-f)}{\left(1+\frac{\tau}{\tau_{3 \mathrm{D}}}\right) \sqrt{1+\frac{\tau}{\tau_{3 \mathrm{D}} \Gamma^{2}}}}+\frac{f}{1+\frac{\tau}{\tau_{2 \mathrm{D}}}}\right)\left(1+\left(\frac{P_{\mathrm{t}}}{1-P_{\mathrm{t}}}\right) \exp \left(\frac{-\tau}{\tau_{\mathrm{t}}}\right)\right),
$$

where $f$ is the fraction of molecules adsorbed onto film; $\tau_{3 \mathrm{D}}$ is the diffusion time of free molecules; $\Gamma$ is the structure parameter of the confocal volume $(4<\Gamma<11$, dependent upon the width of the confocal volume), and was fixed from control measurements of fluorescein dye; and $\tau_{2 \mathrm{D}}$ is the diffusion time of adsorbed molecules. The excitation of electrons into the triplet state is quantified by the triplet lifetime $\tau_{\mathrm{t}}$ and the population of the triplet state, $P_{\mathrm{t}}{ }^{32}, 33$ This model assumes isotropic 2-dimensional diffusion on a homogeneous surface and reduces to the standard bulk autocorrelation function when the confocal volume is moved to the solution (and hence $f=0$ ).

The autocorrelation data were fitted with the above model for times greater than $2 \mu$ s to avoid distortions introduced from afterpulsing in the avalanche detector. The data were analyzed by Levenberg-Marquardt nonlinear least-squares fitting using the pro Fit software package (6.2.9). Surface diffusion coefficients were relatively easy to obtain because the bulk diffusion time was usually well separated from that due to surface diffusion and did not contribute significantly. There was also no observable contribution of free dye in these experiments. Fluorescein has a large diffusion coefficient $\left(>400 \mu \mathrm{m}^{2} \mathrm{~s}^{-1}\right)$ in water. ${ }^{34-36} \mathrm{~N}$ dictated the amplitude of the autocorrelation function and did not interfere with the fitting of $\tau_{2 \mathrm{D}}$. The only other fitting parameter was $f$, which controls the relative contributions of surface and bulk diffusion and was thus easily extracted. Under the conditions of the experiment, $f$ was close to unity. Some diffusion times were measured 
repeatedly in order to obtain the error in the diffusion coefficients. This error was estimated to $\pm 5 \%$ and was independent of temperature.

\section{Friction force microscopy}

A Digital Instruments Dimension 3100 atomic force microscope (AFM) with Nanoscope IV controller (Veeco, Cambridge, UK) was operated in contact mode with a liquid cell/tip holder. Silicon nitride probes (SNL, Bruker, California, USA) with nominal spring constant $0.12 \mathrm{Nm}^{-1}$ and tip radius $2 \mathrm{~nm}$ were used. FFM measurements were performed at a scan rate of $2.03 \mathrm{~Hz}$ with 256 data points per line and with a scan area of $1 \mu \mathrm{m} \times 1 \mu \mathrm{m}$. Each cantilever was calibrated by a Digital Instruments PicoForce module and its associated software, based on the method of Hutter and Bechhoeffer. ${ }^{37}$ confirming the nominal value supplied. The optical lever sensitivity of each modified cantilever was calibrated in DI water at room temperature before each set of experiments. The friction force was acquired by converting the lateral signal collected by the photodetector from voltage to newton using the wedge method, ${ }^{38,39}$ where the cantilever is scanned across a calibration grating (TGF11, MikroMasch, Tallinn, Estonia) and the frictional signal is measured as a function of applied load.

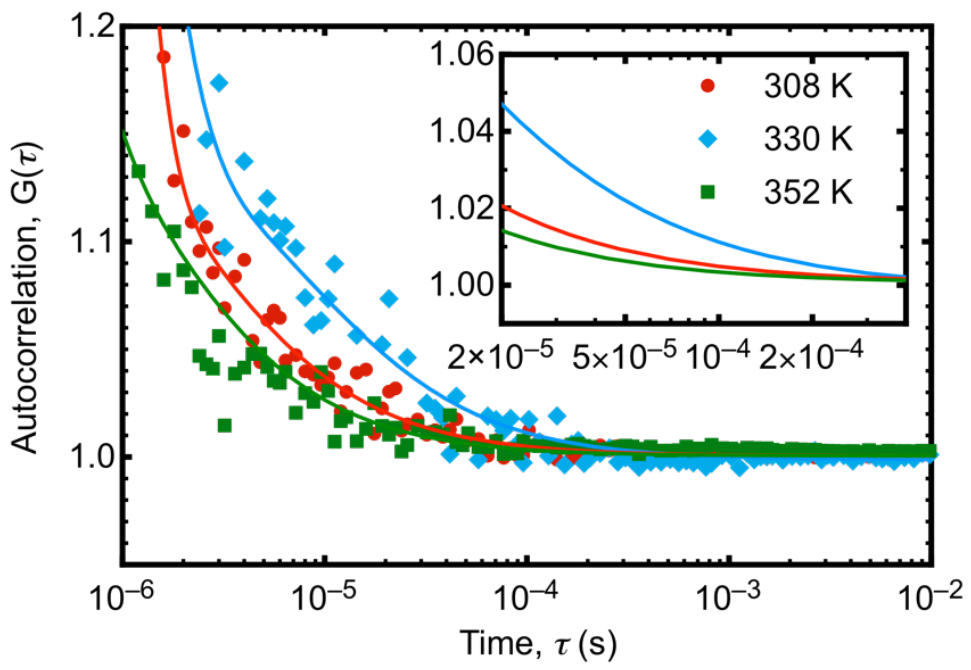

Figure 1: FCS autocorrelation function data and fits for PEO on PEMA films at three different temperatures. The triplet states and bulk diffusion dominate the data at early times, and the shorter (surface) diffusion time of the data at $330 \mathrm{~K}$ is significant from $\tau \approx 10^{-4} \mathrm{~s}$. The fits to the data are shown in the inset, which covers the period where surface diffusion dominates. Here, the autocorrelation function has the steepest decay for the sample measured at $330 \mathrm{~K}$, indicating the shortest diffusion time, and thus the largest diffusion coefficient

\section{RESULTS}

\section{A. Glass transition temperatures}

\begin{tabular}{cccc}
\hline B.Polymer & $T_{\mathrm{gs}}(\mathrm{K})$ & $T_{\mathrm{gb}}(\mathrm{K})$ & $d_{\mathrm{gs}}(\mathrm{nm})$ \\
\hline PMMA & $373 \pm 7$ & $403 \pm 8$ & 252 \\
PEMA & $341 \pm 5$ & $367 \pm 6$ & 282 \\
PPMA & $308 \pm 3$ & $325 \pm 4$ & 352 \\
PBMA & $269 \pm 3$ & $331 \pm 8$ & 279 \\
polystyrene & $356 \pm 4$ & $387 \pm 5$ & 232 \\
\hline
\end{tabular}

Table 1: Glass transition temperatures as measured using ellipsometry 
The glass transition temperatures of films of these polymers was measured by determining the change in expansivity by ellipsometry. ${ }^{5}$ The film temperatures were controlled using a Linkam heating stage under nitrogen flow. The film is taken to comprise a solid and liquid layer with a total thickness dependent upon $T_{\mathrm{gs}}$ and $T_{\mathrm{gb}}$, the surface and bulk glass transition temperatures and their corresponding liquid and solid (glass) expansion coefficients. The film thickness can be fitted using a simple model. ${ }^{40}$ These glass transition temperatures obtained from the ellipsometry data are tabulated in Table 1, which includes $d_{\mathrm{gs}}$, the film thickness at $T=T_{\mathrm{gs}}$.

For the measurement of surface diffusion, fluorescein isothiocyanate end-labeled PEO $(M=20 \mathrm{kDa})$ was allowed to adsorb from a dilute solution in water onto the polymer film, with a clean uncoated silicon wafer used as a control. Diffusion coefficients of PEO were measured using fluorescence correlation spectroscopy (FCS). ${ }^{32}$ Sample FCS data (autocorrelation functions) and fits are shown in Figure 1.
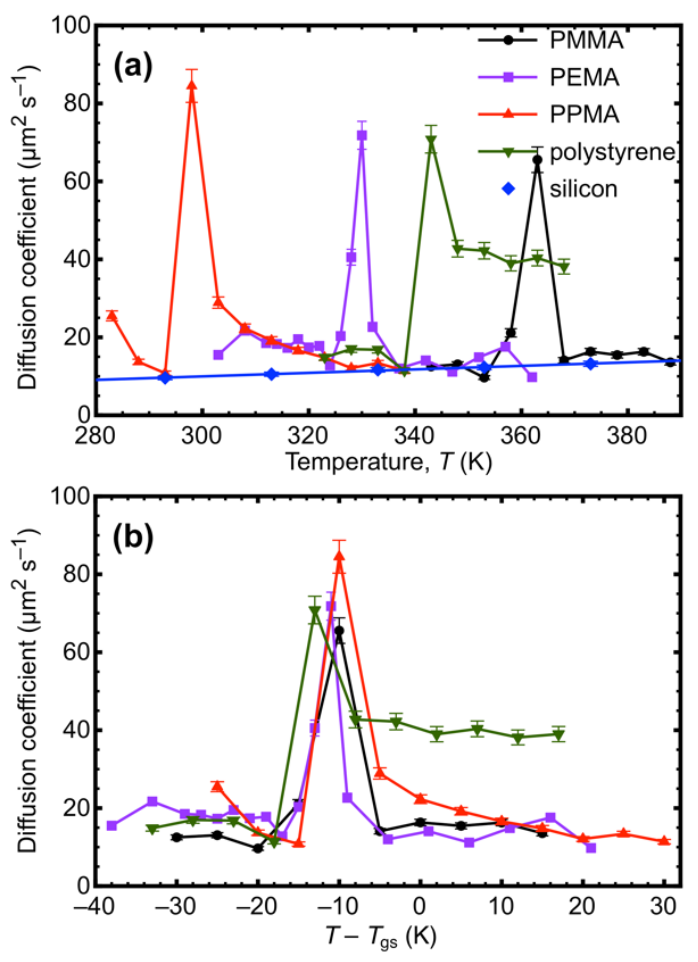

Figure 2: (a) Diffusion profiles of PEO adsorbed onto polymer films for temperatures around their glass transitions. The diffusion coefficients for PEO adsorbed onto bare silicon are also shown. The solid lines are guides to the eye, except for the results for PEO on silicon, for which the solid line is a linear fit to temperature. (b) The same diffusion profiles as in (a), but with the temperature scale relative to the surface glass transition temperature $T_{\mathrm{gs}}$, as determined using ellipsometry

The measured diffusion coefficients are plotted in Figure 2a. The diffusion of PEO on the uncoated silicon wafer follows a simple linear dependence with temperature, as would be expected for Stokes-Einstein behavior. The diffusion of PEO on the different poly(alkyl methacrylate) films also exhibits this behavior, except for temperatures given by $T_{\mathrm{gs}}-T \approx 10 \mathrm{~K}$ (Figure $2 \mathbf{b}$ ), where there is a notable increase in the diffusion coefficient. In addition, PEO was allowed to diffuse on films of blends of PMMA and PBMA, which, being immiscible, exhibit two glass transitions. PBMA and PMMA have a reported interaction parameter of $\chi=$ 0.062 at $T=413 \mathrm{~K}$, based on a lattice size of $0.72 \mathrm{~nm},{ }^{41}$ and are expected to exhibit an upper critical solution temperature, as is the case for PEMA and PMMA. ${ }^{42}$ Diffusion coefficients for PEO on films of blends of PMMA and PBMA are shown in Figure 3. The maximum diffusion coefficients are similar to those in bulk solution for PEO of similar molar mass. ${ }^{22,26,28}$

The blend data reveal two peaks in the diffusion coefficient: one corresponding to the glass transition temperature of the PBMA-rich phase and the other to that of the PMMA-rich phase. In both cases, the peaks 
are located $\sim 10 \mathrm{~K}$ below the corresponding expansivity change measured using ellipsometry (Figure S1b). Only one PEO surface diffusion coefficient was obtained from fitting the blend data at each temperature.
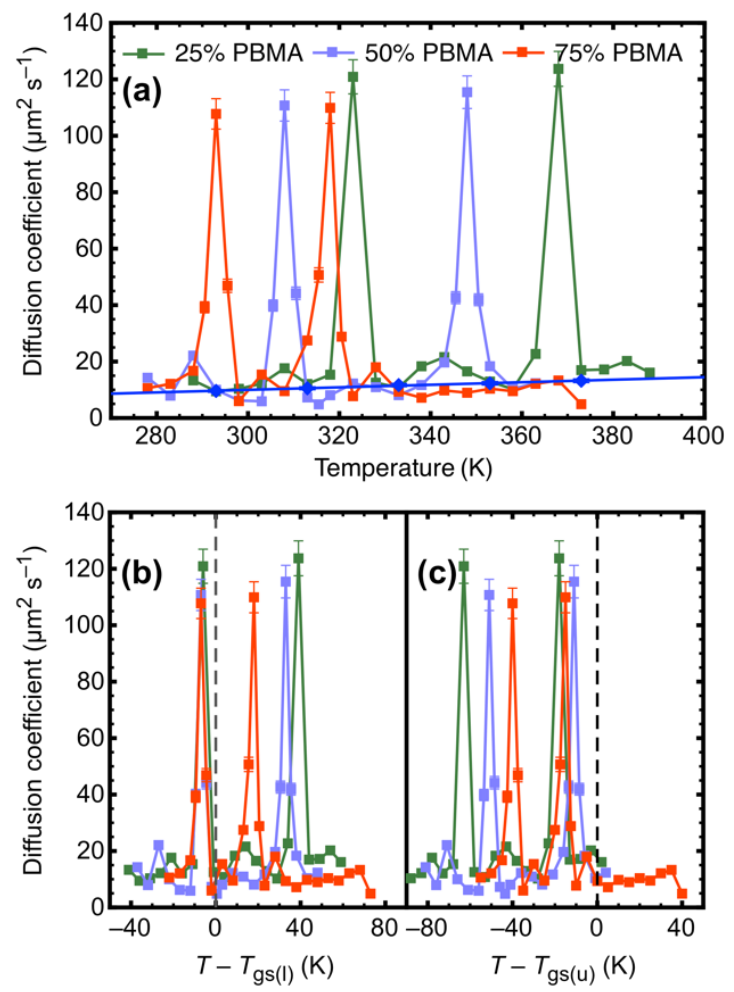

Figure 3: (a) The diffusion of PEO as a function of temperature on blends of PBMA and PMMA revealed two peaks in diffusion coefficient for each blend, indicating two glass transitions. The temperatures are plotted relative to the temperatures of the lower (b), $T_{\mathrm{gs}(1)}$, and upper (c), $T_{\mathrm{gs}(\mathrm{u})}$, transitions, which are indicated by the broken vertical lines

On polystyrene, the PEO also exhibited a separate peak in surface diffusion. In contrast to its behavior on poly(alkyl methacrylate) surfaces, at temperatures greater than those corresponding to the peak in diffusion coefficient, the PEO diffusion on polystyrene did not return to the same behavior exhibited at lower temperatures (Figure 2). It has been well documented that PEO-poly(alkyl methacrylate) blends are at least partially miscible ${ }^{43-46}$ in which case adsorbed PEO has a similar attractive interaction with the poly(alkyl methacrylate) film both above and below $T_{\mathrm{gs}}$. The same does not apply to PEO and polystyrene which are highly immiscible. ${ }^{47}$

In order to directly interrogate the surface of the polymer films, friction force microscopy (FFM) ${ }^{48}$ was performed on a PEMA film under water, as a function of temperature. In Figure 4, FFM data are shown for a PEMA film, with the diffusion coefficient of PEO on the same surface also shown for comparison. The friction coefficient of PEMA increases with temperature above its glass transition, as would be expected for a film close to its melting point. However, again at $T_{\mathrm{gs}}-T \approx 10 \mathrm{~K}$, a narrow peak can be observed. The increase in friction coefficient near, but below, the glass transition temperature of PEMA is unexpected, but more comprehensive studies have revealed the existence of an increase in frictional behavior close to the glass transition in polystyrene films. ${ }^{49}$ The FFM set-up used in this work can only be operated in a restricted temperature range, so these experiments were restricted to PEMA films.

\section{DISCUSSION}

The large increase in diffusion coefficient indicates a PEO conformational transition at $T=T_{\mathrm{gs}}-10 \mathrm{~K}$. PEO fully adsorbed onto a substrate (pancake structure) has a diffusion coefficient an order of magnitude smaller than that when it takes up a conformation comprising loops and trains, ${ }^{23}$ so it can be concluded that the surface of the polymer film triggers a conformational change in the PEO. However, it is not likely that PEO has a flat (pancake) structure on these films; previous measurements have indicated that when it has a pancake 
structure, $(20 \mathrm{kDa})$ PEO diffuses with a coefficient close to $0.1 \mu \mathrm{m}^{2} / \mathrm{s}$, ${ }^{22}$ which is smaller than the values measured here. PEO is also reticent to adsorb to PMMA, silica, or polystyrene. ${ }^{50}$ That PEO takes up a pancake structure has been questioned for hydrophobic surfaces ${ }^{51}$ but force spectroscopy has revealed that adsorption as a pancake does occurs on some surfaces. ${ }^{23}$ Bulk measurements of PEO diffusion have a diffusion coefficient similar to the maximum surface diffusion values reported here, ${ }^{22,26,28}$ and so it may be argued that the conformational transition just below the glass transition amounts to desorption. Nevertheless, the relative contributions of adsorption and desorption are important in some diffusion mechanisms. ${ }^{52,53}$

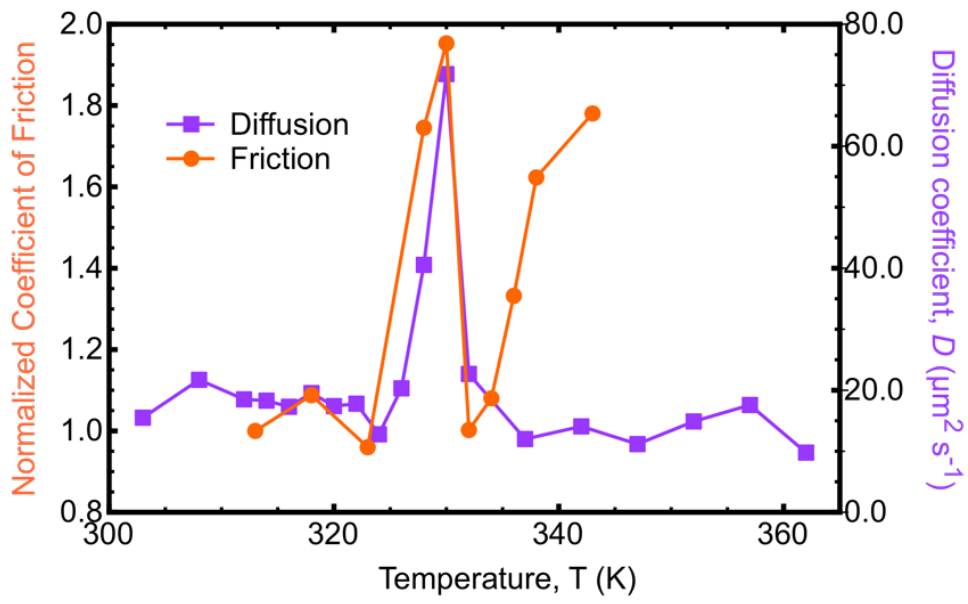

Figure 4: FFM data reveal that a peak in the coefficient of friction of a silicon nitride AFM tip on a PEMA surface. The friction data are normalized to the result at $313 \mathrm{~K}$

Both poly(alkyl methacrylate) films and the native oxide layer of silicon surfaces offer hydrogen bonding sites with which the PEO can interact. In order to test the effect of nonpolar surfaces on the diffusion, polystyrene films were used. As can be seen in Figure 2a, there is again a peak in the diffusion coefficient at $T \approx T_{\mathrm{gs}}-10 \mathrm{~K}$. However, at greater temperatures, the diffusion coefficient does not return to values of the silicon or the poly(alkyl methacrylate) films but remains at a large value, albeit below the maximum. It is known however, that PEO undergoes a conformational transition at temperatures greater than its $\theta$ temperature $)^{54}$ and that its adsorption to polystyrene is affected. ${ }^{55}$ The related possibility that the more polar poly(alkyl methacrylate) films might absorb some water deserves some consideration. However, the finding that PEO exhibits a peak in its diffusion coefficient on both polystyrene and poly(alkyl methacrylate) films at $\sim 10 \mathrm{~K}$ below the surface glass transition shows that the swelling of these films by water is not a significant effect. Earlier neutron reflectometry experiments have also shown limited water swelling of glassy PMMA and rubbery PBMA films. ${ }^{56}$

A recent report has shown that tobacco mosaic virus (TMV) diffuses on the surface of a glassy film of $N, N^{\prime}$ bis(3-methylphenyl)- $N, N^{\prime}$-diphenylbenzidine independently of film thickness, despite the observed film thickness dependence of the glass transition temperature. ${ }^{57}$ The conclusion from this work was that, near the glass transition, there was a discrete layer of greater segmental mobility, the properties of which were independent of the underlying film. This conclusion is consistent with the present results. Nevertheless, the limited temperature dependence of the TMV surface diffusion experiments do not allow a direct comparison. Furthermore, the TMV is large and asymmetric ${ }^{58}$ and perhaps not an ideal comparator.

As can be seen in Figure 4, there is a peak in the friction coefficient at a similar temperature to that in the diffusion coefficient (also shown). A proxy for temperature is that of time, and by changing the scanning rate it has been shown elsewhere that the glass transition has an effect on the time-temperature superposition coefficient at the glass transition for polystyrene. ${ }^{49}$ Other experiments performed using AFM to determine mechanical properties of nanoparticle-embedded polystyrene also provide evidence for a shift in behavior in the vicinity of the glass transition, where evidence of surface stiffening was found. ${ }^{59}$ 
Ultimately, a sharp AFM tip interacting with the polymer surface may be of comparable size to $20 \mathrm{kDa}$ PEO and it is possible that the size of the object which perturbs the film is relevant. As has been pointed out, ${ }^{30} \mathrm{a}$ thin layer has associated capillary waves that have a dominant wavelength $\lambda \approx 2 \pi h$, where $h$ is a film thickness. Longer wavelengths will be impeded by the thickness of the layer and shorter ones will be thermodynamically suppressed because they create too much interface. The tantalizing suggestion that a dominant mode couples to the adsorbed polymer can only be tested by a comprehensive study covering a large range of molar masses.

\section{CONCLUSIONS}

To summarize, data have been presented to show that the nature of the surface of the film has a profound effect on the diffusion of polymers adsorbed on that film. At temperatures some $10 \mathrm{~K}$ below the surface glass transition an anomalous peak in the diffusion coefficient is observed, the origin of which is unclear, but it may be speculated that the adsorbed PEO is interacting with a nanoscale surface layer. This behavior is observed for PEO adsorbed on polystyrene films and three poly(alkyl methacrylate) films, as well as blends of PBMA and PMMA, and may therefore be considered to be a general phenomenon.

\section{Supplementary materials}

Ellipsometry thickness results; FCS hysteresis data and results for the diffusion of dextran on PEMA, which also show a diffusion maximum; water contact angle analysis; and scanning force and optical microscopy images of blend films.

\section{Data availability statement}

The data that support the findings of this study are available from the first authors upon reasonable request. Raw FCS data are available on the White Rose Repository.

\section{Acknowledgments}

The EPSRC is acknowledged for providing financial support through a doctoral training award for MM and through EP/I012060/1 for ZZ. David Spiller at the University of Manchester is acknowledged for help with the FCS experiments of dextran presented in the supporting information.

\section{Present addresses}

ZJZ: School of Chemical Engineering, University of Birmingham, Birmingham B15 2TT, UK

RCDJ: Department of Chemistry, Durham University, South Road, Durham DH1 3LE, UK

YS: School of Physics and Astronomy, University of Edinburgh, Edinburgh EH9 3FD, UK

TJBB: Department of Chemistry, Imperial College London, White City Campus, London W12 7TA, UK

MG: School of Engineering, Newcastle University, Merz Court, Newcastle-upon-Tyne NE1 7RU, UK

(mark.geoghegan@newcastle.ac.uk)

\section{REFERENCES}

${ }^{1}$ M. Alcoutlabi, and G. B. McKenna, J. Phys.: Condens. Matter 17 (2005) R461.

2 M. D. Ediger, and J. A. Forrest, Macromolecules 47 (2014) 471.

${ }^{3}$ S. Napolitano, E. Glynos, and N. B. Tito, Rep. Prog. Phys. 80 (2017) 036602.

${ }^{4}$ K. S. Schweizer, and D. S. Simmons, J. Chem. Phys. 151 (2019) 240901.

5 J. L. Keddie, R. A. L. Jones, and R. A. Cory, Europhys. Lett. 27 (1994) 59.

${ }^{6}$ J. L. Keddie, R. A. L. Jones, and R. A. Cory, Faraday Discuss. 98 (1994) 219.

7 A. Debot, R. P. White, J. E. G. Lipson, and S. Napolitano, ACS Macro Lett. 8 (2019) 41.

${ }^{8}$ R. P. White, and J. E. G. Lipson, Macromolecules 49 (2016) 3987.

${ }^{9}$ G. Reiter, Europhys. Lett. 23 (1993) 579.

${ }^{10}$ R. L. Jones, S. K. Kumar, D. L. Ho, R. M. Briber, and T. P. Russell, Macromolecules 34 (2001) 559.

11 A. Silberberg, J. Colloid Interface Sci. 90 (1982) 86.

${ }^{12}$ A. Brûlet, F. Boué, A. Menelle, and J. P. Cotton, Macromolecules 33 (2000) 997.

${ }^{13}$ M. Geoghegan, and G. Krausch, Prog. Polym. Sci. 28 (2003) 261.

${ }^{14}$ N. Rehse, C. Wang, M. Hund, M. Geoghegan, R. Magerle, and G. Krausch, Eur. Phys. J. E 4 (2001) 69.

${ }^{15}$ C. J. Ellison, and J. M. Torkelson, Nature Mater. 2 (2003) 695.

${ }^{16}$ C. B. Roth, K. L. McNerny, W. F. Jager, and J. M. Torkelson, Macromolecules 40 (2007) 2568.

${ }^{17}$ Z. Yang, A. Clough, C.-H. Lam, and O. K. C. Tsui, Macromolecules 44 (2011) 8294.

${ }_{18}$ V. M. Boucher, D. Cangialosi, H. Yin, A. Schönhals, A. Alegria, and J. Colmenero, Soft Matter 8 (2012) 5119.

${ }^{19}$ J. A. Forrest, and K. Dalnoki-Veress, ACS Macro Lett. 3 (2014) 310. 
${ }^{20}$ C. Zhang, Y. Guo, and R. D. Priestley, Macromolecules 44 (2011) 4001.

${ }^{21}$ M. Mears, D. S. Tarmey, and M. Geoghegan, Macromol. Rapid Commun. 32 (2011) 1411.

22 S. A. Sukhishvili, Y. Chen, J. D. Müller, E. Gratton, K. S. Schweizer, and S. Granick, Nature 406 (2000) 146.

${ }^{23}$ P. Burgos, Z. Zhang, R. Golestanian, G. J. Leggett, and M. Geoghegan, ACS Nano 3 (2009) 3235.

${ }^{24}$ G. T. Morrin, and D. K. Schwartz, Macromolecules 51 (2018) 1207.

${ }^{25}$ D. Mukherji, and M. H. Müser, Phys. Rev. E 74 (2006) 010601(R).

${ }^{26}$ Z. J. Zhang, S. Edmondson, M. Mears, J. Madsen, S. P. Armes, G. J. Leggett, and M. Geoghegan, Macromolecules 51 (2018) 6312.

${ }^{27}$ J. Zhao, and S. Granick, J. Am. Chem. Soc. 126 (2004) 6242.

${ }^{28}$ C. G. Clarkson, A. Johnson, G. J. Leggett, and M. Geoghegan, Nanoscale 11 (2019) 6052.

${ }^{29}$ D. Wang, C. He, M. P. Stoykovich, and D. K. Schwartz, ACS Nano 9 (2015) 1656.

${ }^{30}$ S. Herminghaus, R. Seemann, and K. Landfester, Phys. Rev. Lett. 93 (2004) 017801.

${ }^{31}$ E. L. Elson, and D. Magde, Biopolymers 13 (1974) 1.

${ }^{32}$ E. Haustein, and P. Schwille, Annu. Rev. Biophys. Biomol. Struct. 36 (2007) 151.

33 J. Widengren, Ü. Mets, and R. Rigler, J. Phys. Chem. 99 (1995) 13368.

${ }_{34}$ C. T. Culbertson, S. C. Jacobson, and J. M. Ramsey, Talanta 56 (2002) 365.

35 P. H. Paul, M. G. Garguilo, and D. J. Rakestraw, Anal. Chem. 70 (1998) 2459.

36 Z. Petrášek, and P. Schwille, Biophys. J. 94 (2008) 1437.

${ }^{37}$ J. L. Hutter, and J. Bechhoefer, Rev. Sci. Instrum. 64 (1993) 1868.

${ }_{38}$ D. F. Ogletree, R. W. Carpick, and M. Salmeron, Rev. Sci. Instrum. 67 (1996) 3298.

${ }^{39}$ M. Varenberg, I. Etsion, and G. Halperin, Rev. Sci. Instrum. 74 (2003) 3362.

40 J. E. G. Lipson, and S. T. Milner, Macromolecules 43 (2010) 9874.

${ }^{41}$ J. Scherble, B. Stark, B. Stühn, J. Kressler, H. Budde, S. Höring, D. W. Schubert, P. Simon, and M. Stamm, Macromolecules 32 (1999) 1859.

42 J. A. Schroeder, F. E. Karasz, and W. J. MacKnight, Polymer 26 (1985) 1795.

${ }^{43}$ R. H. Colby, Polymer 30 (1989) 1275.

${ }^{44}$ M. Dionísio, A. C. Fernandes, J. F. Mano, N. T. Correia, and R. C. Sousa, Macromolecules 33 (2000) 1002.

${ }^{45}$ E. E. Shafee, and W. Ueda, Eur. Polym. J. 38 (2002) 1327.

${ }^{46}$ J. A. Zawada, C. M. Ylitalo, G. G. Fuller, R. H. Colby, and T. E. Long, Macromolecules 25 (1992) 2896.

${ }^{47}$ E. Yilmaz, O. Yilmaz, and H. Caner, Eur. Polym. J. 32 (1996) 927.

${ }^{48}$ R. W. Carpick, and M. Salmeron, Chem. Rev. 97 (1997) 1163.

${ }^{49}$ T. Kajiyama, K. Tanaka, N. Satomi, and A. Takahara, Macromolecules 31 (1998) 5150.

${ }^{50}$ C. Chen, M. A. Even, J. Wang, and Z. Chen, Macromolecules 35 (2002) 9130.

${ }_{51}$ M. J. Skaug, J. N. Mabry, and D. K. Schwartz, J. Am. Chem. Soc. 136 (2014) 1327.

52 O. V. Bychuk, and B. O’Shaughnessy, J. Chem. Phys. 101 (1994) 772.

${ }^{53}$ D. Wang, and D. K. Schwartz, J. Phys. Chem. C 124 (2020) 19880.

${ }^{54}$ C. Ö. Dïnç, G. Kïbarer, and A. Güner, J. Appl. Polym. Sci. 117 (2010) 1100.

${ }_{55}$ T. Kato, K. Nakamura, M. Kawaguchi, and A. Takahashi, Polym. J. 13 (1981) 1037.

${ }^{56}$ C. White, K. T. Tan, D. Hunston, K. Steffens, D. L. Stanley, S. K. Satija, B. Akgun, and B. D. Vogt, Soft Matter 11 (2015) 3994.

${ }^{57}$ Y. Zhang, and Z. Fakhraai, Proc. Natl Acad. Sci. USA 114 (2017) 4915.

58 W. M. Stanley, and T. F. Anderson, J. Biol. Chem. 139 (1941) 325.

59 T. B. Karim, and G. B. McKenna, Polymer 54 (2013) 5928. 


\section{Supplementary Information for:}

\section{Lateral diffusion of single poly(ethylene oxide) chains on the surfaces of glassy and molten polymer films}

Matthew Mears, Zhenyu J. Zhang, Ryan C. D. Jackson, Yuchen Si, Tigerlily J. B. Bradford, John M. Torkelson, and Mark Geoghegan ${ }^{1}$

\section{Ellipsometry}
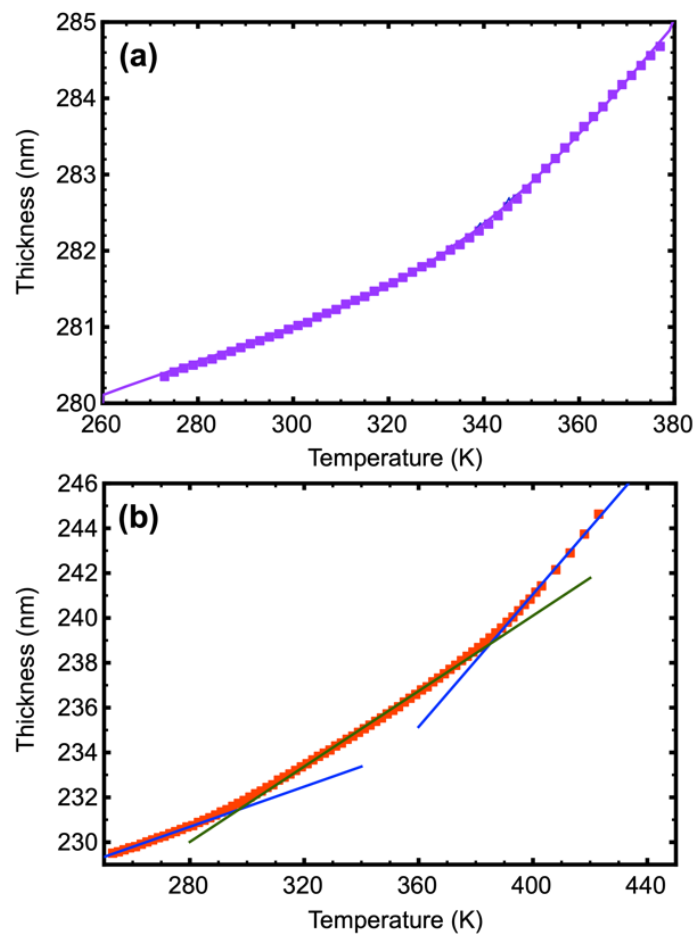

Figure S1: Ellipsometry data for (a) a PEMA film, and (b) a blend of 75\% PBMA in PMMA. In the case of the blend, the two expansivities correspond to the different glass transition temperatures

The usual method for measuring the glass transition using ellipsometry ${ }^{1}$ accounts for the thermal expansivity of the film being different in the glassy phase compared to the liquid phase. The thickness increases linearly with temperature at the highest and lowest temperatures measured, but between the surface and bulk glass transitions, the thickness must be modeled. ${ }^{2}$ Example data for a poly(ethyl methacrylate) (PEMA) film are shown in Figure S1a and for a blend containing 75\% by weight poly(butyl methacrylate) (PBMA) mixed with poly(methyl methacrylate) (PMMA) in Figure S1b.

$\begin{array}{lcc} & \text { Glass }\left(\mathrm{pm} \mathrm{K}^{-1}\right) & \text { Liquid }\left(\mathrm{pm} \mathrm{K}^{-1}\right) \\ \text { PBMA } & 90 & 190 \\ \text { PPMA } & 101 & 232 \\ \text { PEMA } & 81 & 186 \\ \text { PMMA } & 54 & 154\end{array}$

Table S1: Thermal expansivities as measured by ellipsometry. Random errors in the measurement are no more than 1 pm K-1.

The coefficients of thermal expansion for the different poly(alkyl methacrylate) films as measured by ellipsometry are included in Table S1. The value of PMMA presented here are a little larger than those for 
PMMA on gold, ${ }^{3}$ and those for PBMA and PEMA are in moderately good agreement with other measurements. ${ }^{4}$

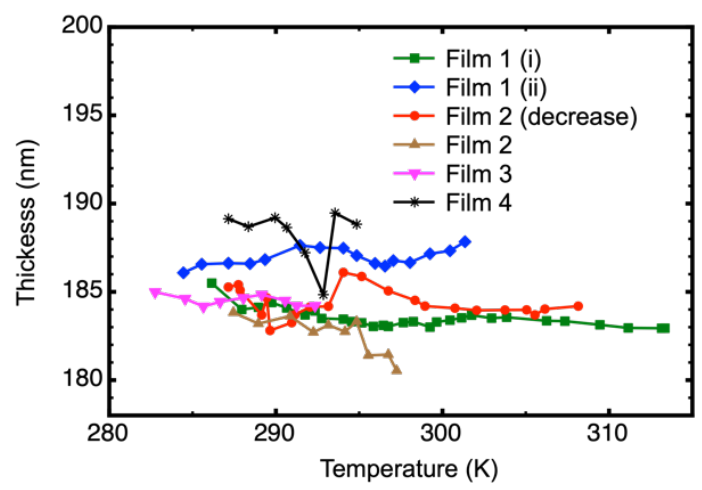

Figure S2: Ellipsometry data four different PBMA films immersed in water as a function of increasing temperature, except where indicated. Film 1 was measured twice, with the second measurement exhibiting a greater thickness

The thickness of films of PBMA and PEMA were measured using ellipsometry in water. Here, films were placed in a sample cell with the polarized light from the ellipsometer (Woollam alpha-SE) entering through quartz windows. The cell was immersed in water and cooled using ice. A resistive heating element was used to increase the temperature, and the cell was sealed with a metal cover. A thermocouple was used to monitor the temperature. Both PEMA and PBMA swelled slightly in water compared to their dry thickness. Nevertheless, the experimental setup here was unable to reveal any thickness changes due to a glass transition. In Figure S2 data for PBMA films are shown.

\section{Temperature hysteresis in diffusion and contact angle data}

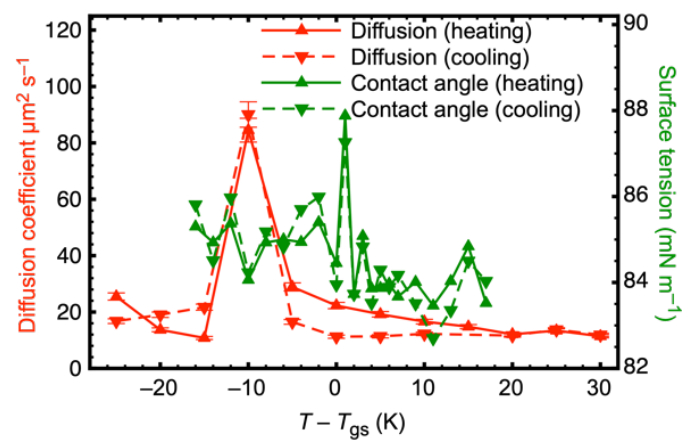

Figure S3: FCS and contact angle results for PEO diffusion on a PPMA film. Experiments were performed with heating preceding a cooling run

All substrates were prepared in the manner described in the Experimental section and were heated to well above their respective glass transitions to allow the chains to relax. After cooling at a constant rate to the initial measurement temperature, the films were mounted onto the measurement stage of a Theta optical tensiometer (Attension, Biolin Scientific, Espoo, Finland) including a fixed Linkam heating platform (Linkam Scientific Instruments Ltd) with TMS94 heat controller and LNP-1 nitrogen flow control. All measurements were performed using Milli-Q purified water as the liquid phase component, and surface tensions were calculated using the included software averaging over 300 frames. The temperature-dependent water contact angle on poly(propyl methacrylate) (PPMA) films is shown in Figure S3.

In order to determine whether temperature hysteresis effects occur, a test was performed whereby the sample was first heated above the glass transition, then brought down to room temperature. This was performed for both poly(ethylene oxide) (PEO) diffusion (fluorescence correlation spectroscopy, FCS) or surface tension measurements. As can be seen from the results (Figure S3), there is no effect of temperature hysteresis in either of these experiments. The temperature was first increased and then reduced, and the diffusion coefficient and surface tension were the same for the cooling cycle as that measured during heating. A change 
(peak) in the contact angle was observed, but at $T=T_{\mathrm{gs}}$. Although measurements of the advancing and receding contact angle hysteresis would provide more detail on the temperature-dependent hysteresis, these static measurements convincingly demonstrate that, like the diffusion measurements, it is unimportant whether the temperature is increasing or decreasing. It is curious that the peaks revealed by macroscopic water droplets and the nanoscopic diffusion and friction force data are observed at different temperatures.

\section{Microscopy of blends}

Scanning force microscopy (SFM) was performed on a 23\% mixture (by weight) of PBMA in poly(methyl methacrylate) (PMMA) in air. Here (Figure S4) one can see that there is a relatively large height difference between the highest and lowest point of the film of $\sim 15 \mathrm{~nm}$, which is believed to be due to phase separation below the surface of the film. An optical microscope image of a similar sample before annealing revealed large-scale phase separation in the as-cast film (Figure S5). Films annealed at $393 \mathrm{~K}$ for $1 \mathrm{~h}$ show similar sized regions of separations, indicating that phase separation remains consistent over the timescales for FCS, ellipsometry and contact angle experiments.

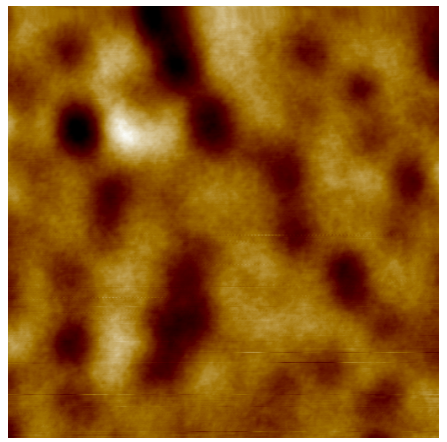

Figure S4: SFM data for a blend film of 23\% PBMA with PMMA. The sample was annealed at a temperature of $393 \mathrm{~K}$ for $1 \mathrm{~h}$. The width of the image is $5 \mu \mathrm{m}$ and the height scale is $15 \mathrm{~nm}$. This image was taken using tapping mode in air

For the optical measurements, films were imaged using a Nikon Eclipse E600 microscope (Nikon Limited UK, Surrey, UK) in reflection mode at 50× magnification. All homopolymer films were uniform, whereas blend films showed regions of phase separation. Scanning force microscopy data were obtained using a Digital Instruments Dimension 3100 atomic force microscope with Nanoscope IV controller (Veeco, Cambridge, UK) operating in tapping mode using SNL probes.

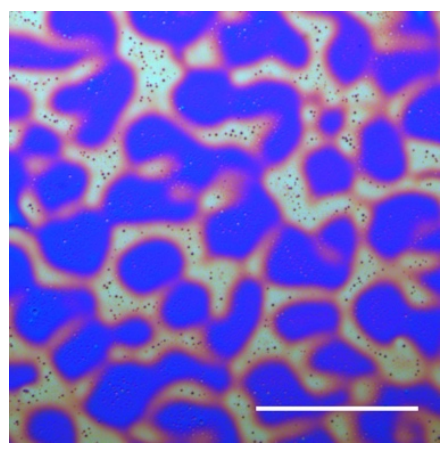

Figure S5: Optical microscope image of a blend of 23\% PBMA in PMMA film before annealing. The scale bar represents $50 \mu \mathrm{m}$ 


\section{Diffusion of dextran}

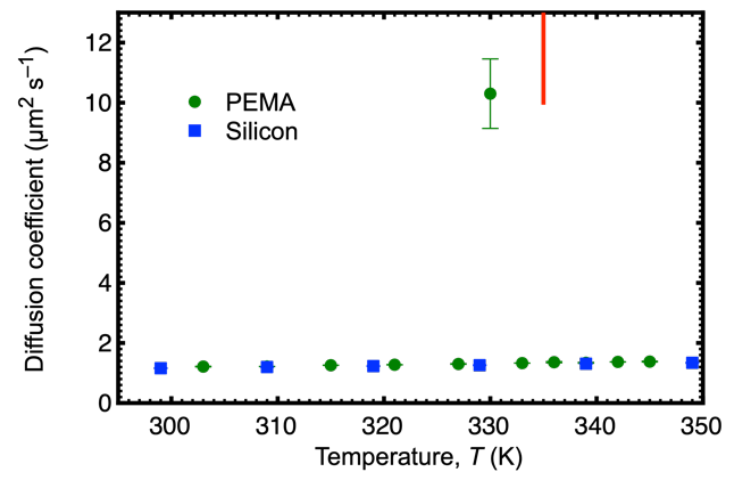

Figure S6: FCS two-dimensional diffusion coefficients for the diffusion of dextran on PEMA and silicon. The red line corresponds to the surface glass transition of this PEMA film $\left(T_{\mathrm{gs}}=335 \pm 7 \mathrm{~K}\right)$ as measured by ellipsometry

In order to see whether or not the observation of an anomalous peak in surface diffusion on polymer surfaces at temperatures below their thin film glass transition was specific to PEO, experiments were also performed with dextran. The diffusion of fluorescein isothiocyanate-labeled dextran $(20 \mathrm{kDa}$, purchased from SigmaAldrich) was measured using a Zeiss LSM 780 with ConfoCor 3 FCS module at the University of Manchester Systems Microscopy Centre. The diffusion coefficients of dextran on PEMA and silicon surfaces are shown in Figure S6. Again, there is a peak in the diffusion coefficient confirming that the PEO results are not unique. Given the different polymers used for the diffusing species and the films, the phenomenon it can be concluded that the phenomenon is general and provides important insight into the glass transition of polymer films.

${ }^{1}$ J. L. Keddie, R. A. L. Jones, and R. A. Cory, Europhys. Lett. 27 (1994) 59.

2 J. E. G. Lipson, and S. T. Milner, Macromolecules 43 (2010) 9874.

${ }^{3}$ J. L. Keddie, R. A. L. Jones, and R. A. Cory, Faraday Discuss. 98 (1994) 219.

${ }^{4}$ ThetaMetrisis Application Note \#013 ThetaMetrisis LLC (Redwood City CA, 2014) 